\title{
BMJ Open Volume and in-hospital mortality after emergency abdominal surgery: a national population-based study
}

\author{
Deirdre M Nally (D) , ${ }^{1}$ Jan Sørensen, ${ }^{2}$ Gintare Valentelyte (D) , ${ }^{2}$ Laura Hammond, ${ }^{2,3}$ \\ Deborah McNamara, ${ }^{3}$ Dara O Kavanagh, ${ }^{1}$ Ken Mealy ${ }^{3}$
}

To cite: Nally DM, Sørensen J Valentelyte $\mathrm{G}$, et al. Volume and in-hospital mortality after emergency abdominal surgery: a national populationbased study. BMJ Open 2019;9:e032183. doi:10.1136/ bmjopen-2019-032183

- Prepublication history and additional material for this paper are available online. To view, please visit the journal (http:// dx.doi.org/10.1136/bmjopen2019-032183).

Received 06 June 2019 Revised 16 August 2019 Accepted 07 October 2019

Check for updates

(c) Author(s) (or their employer(s)) 2019. Re-use permitted under CC BY-NC. No commercial re-use. See rights and permissions. Published by BMJ.

${ }^{1}$ Department of Surgical Affairs, Royal College of Surgeons in Ireland (RCSI), Dublin, Ireland

${ }^{2}$ Healthcare Outcomes Research Centre, Royal College of Surgeons in Ireland (RCSI), Dublin, Ireland

${ }^{3}$ National Clinical Programme in Surgery (NCPS), Royal College of Surgeons (RCSI), Dublin, Ireland

Correspondence to

Dr Deirdre M Nally;

deirdrenally@rcsi.com

\section{ABSTRACT}

Objectives Emergency abdominal surgery (EAS) refers to high-risk intra-abdominal surgical procedures undertaken for acute gastrointestinal pathology. The relationship between hospital or surgeon volume and mortality of patients undergoing EAS is poorly understood. This study examined this relationship at the national level.

Design This is a national population-based study using a full administrative inpatient dataset (National Quality Assurance Improvement System) from publicly funded hospitals in Ireland.

Setting 24 public hospitals providing EAS services. Participants and Interventions Patients undergoing EAS as identified by primary procedure codes during the period 2014-2018.

Main outcome measures The main outcome measure was adjusted in-hospital mortality following EAS in publicly funded Irish hospitals. Mortality rates were adjusted for sex, age, admission source, Charlson Comorbidity Index, procedure complexity, organ system and primary diagnosis. Differences in overall, 7-day and 30-day inhospital mortality for hospitals with low $(<250)$, medium (250-449) and high (450+) volume and surgical teams with low $(<30)$, medium $(30-59)$ and high $(60+)$ volume during the study period were also estimated.

Results The study included 10344 EAS episodes. 798 in-hospital deaths occurred, giving an overall in-hospital mortality rate of 77 per 1000 episodes. There was no statistically significant difference in adjusted mortality rate between low and high volume hospitals. Low volume surgical teams had a higher adjusted mortality rate (85.4 deaths/1000 episodes) compared with high volume teams (54.7 deaths/1000 episodes), a difference that persisted among low volume surgeons practising in high volume hospitals.

Conclusion Patients undergoing EAS managed by high volume surgeons have better survival outcomes. These findings contribute to the ongoing discussion regarding configuration of emergency surgery services and emphasise the need for effective clinical governance regarding observed variation in outcomes within and between institutions.

\section{INTRODUCTION}

Emergency abdominal surgery (EAS) refers to a variety of intra-abdominal surgical procedures undertaken for acute gastrointestinal

\section{Strengths and limitations of this study}

- This study analyses a complete national emergency abdominal surgery (EAS) database over a contemporary 5-year period.

- The publicly funded EAS service is available to all residents independent of financial means.

- This reduces the variation in access observed in other settings and increases generalisability of the findings.

- The limitations of this study are those inherent to the interpretation of all administrative databases including the accuracy of coding performed at hospital level.

- The database used for this study (National Quality Assurance Improvement System) consists of administrative data captured at the time of discharge; it is not linked to death registries thus, it captures only in-hospital deaths and hence, probably underestimates overall mortality.

conditions. It is high-risk surgery, associated with significant mortality and morbidity. Outcomes are poorest for older, frail patients. ${ }^{1}$ Inferior outcomes are also experienced by patients from ethnic minorities, lower socioeconomic groups and those who are uninsured. ${ }^{2} 3$

In high-risk elective surgery, identification of an association between hospital and surgeon volume and outcomes has prompted many improvements including the reconfiguration of elective surgical services. ${ }^{4}$ In contrast, despite greater risks associated with EAS, ${ }^{5}{ }^{6}$ relationships between volume and outcomes in emergency surgery are poorly understood. Patients presenting for EAS are a vulnerable group as the acute nature of symptoms requires urgent care, resulting in less choice of hospital and surgeon provider. Thus, the provision of high-quality and safe EAS is an important public health concern.

EAS outcomes depend on patient and surgical system factors. Multicentre, national and international studies on EAS outcomes 
have been published from the UK, ${ }^{7}$ Denmark, ${ }^{9-11}$ Australia, ${ }^{12}{ }^{13}$ the USA, ${ }^{714}$ Canada $^{1}$ and South Africa. ${ }^{15}$ These studies promoted safer EAS care through the introduction of clinical pathways and sustainable national audits. There is, however, heterogeneity between studies with respect to case selection limiting direct comparison. Furthermore, these studies examined selected hospitals or geographical regions which limit generalisation to an entire population. In the Ireland, health services are organised such that almost all EAS is performed in the public health service and captured in a single national database, enabling insight into EAS outcomes at a population level. Our objective was to determine mortality following EAS at the national level and to investigate the relationship between volume and mortality. Specifically, this study examines the relationship between hospital volume and surgeon volume and in-hospital mortality. Improved understanding of this relationship can inform policy decisions regarding the structure of EAS at regional and national levels.

\section{METHODS}

\section{Healthcare context}

Publicly funded healthcare in Ireland (population 4.8 million) is available to all residents. It is organised and delivered by the Health Service Executive. Public hospitals are divided into seven hospital groups with geographically defined catchment areas. Each group has at least one designated cancer centre and a number of other hospitals, classified into four levels ('models') with increasing complexity of care available at each level. Regarding acute surgical services, model 3 hospitals provide care to undifferentiated surgical patients; model 4 hospitals provide acute undifferentiated care and accept tertiary referrals. ${ }^{16}$ Twenty-four hospitals provide acute surgical services, each operating an on-call rota to provide emergency services on a 24-hour basis. In each hospital, data from patient medical records are coded by trained coders and submitted to the national Hospital In-Patient Enquiry (HIPE) System. The National Quality Assurance Improvement System (NQAIS) is a data extraction system by which HIPE data can be retrieved and analysed. This national, population-based study using NQAIS data is reported according to REporting of studies Conducted using Observational Routinely-collected health Data Guidelines (online supplementary appendix 1 ), ${ }^{17}$ an extension to the Strengtheningthe Reporting of Observational Studies in Epidemiology guidelines. ${ }^{18}$

\section{Data extraction}

Each HIPE discharge record holds demographic, clinical and administrative data for completed inpatient episodes. Procedures and diagnoses are coded according to the Australian Classification of Health Interventions (8th edition) and the International Classification of Diseases, 10th Revision, Australian Modification (2013). ${ }^{19}$
Procedure codes, identified by the authors as representing EAS, were defined. These procedures are listed in online supplementary appendix 2. Appendicectomy, cholecystectomy, aortic and trauma surgical emergencies were excluded. Data relating to all emergency discharges during the study period, 1 January 2014-31 December 2018, where EAS was the primary procedure, was extracted from NQAIS. One hospital was excluded from analysis as EAS was performed only during the first 2 years of the study period; EAS was then transferred to another included hospital. Only episodes relating to patients aged $>16$ years, coded as an emergency inpatient admission, were included in the analysis.

For each discharge the following variables were available: age, sex, Charlson Comorbidity Index (CCI), primary procedure code, primary and secondary diagnoses, dates of admission, discharge and primary procedure, hospital name, admission type and source, discharge destination, possible transfer details and team codes for the principal and procedure surgical team.

The primary outcome measure was overall in-hospital mortality. Further analysis was performed on in-hospital mortality within 7 and 30 days of admission and surgery. The HIPE database is not linked to national death registries, therefore mortality posthospital discharge was not available.

\section{Categorisation}

In order to examine the relationship between hospital volume and in-hospital mortality, hospital volume was categorised into low, medium and high volumes based on consideration of the data and the healthcare context: hospitals were ranked by the total number of episodes of care during the study period. The 25th and 75th percentile volumes were identified as cut-off points. One of the eight designated national cancer centres was re-categorised from the medium to the high volume group to ensure that all specialist regional centres were represented in this grouping. Low volume hospitals were defined as those that had performed $<250$ procedures, medium volume hospitals performed 250-449 procedures and high volume hospitals performed 450 or more procedures.

HIPE experts and individual hospital HIPE coders were consulted to clarify surgical team coding principles. The term 'surgical team' refers to an individual consultant surgeon and associated clinical team. For surgeon volume analysis, only episodes where the principal surgical team code and the procedure team code were identical and the code referred to a consultant general surgeon were included. This safeguards the validity of this volume analysis. Surgical team volume was defined according to the number of procedures performed during the study period: low, medium and high volume teams performed $<30,30-59$ or 60 or more procedures, respectively. 


\section{Analysis}

Variation in in-hospital mortality associated with volume was assessed. There was no substantial variation in case-mix, crude or adjusted in-hospital mortality rates in the single years (online supplementary table $\mathrm{S} 1$ ). Thus, to increase statistical power, the 5 years of data were analysed together. We estimated the adjusted overall in-hospital mortality rate and mortality rates 7 and 30 days after the date of admission and after the date of the primary operative procedure (sensitivity analysis in the online material supplementary table 2).

Poisson regression was used to estimate standardised mortality rates. Results are reported in the form of marginal estimates from the Poisson regressions and presented as adjusted rates with 95\% CIs. To standardise, we included categorical variables for sex, 10-year age group, admission source, CCI category, procedure complexity, organ system and primary diagnosis. Preliminary analyses tested a range of other variables including sex, age, admission source (home, other hospital, nursing home), procedure complexity, insurance status and medical card status (an entitlement to free public health service based on health or socioeconomic indicators). These factors did not improve the model fit. To account for potential clustering effects (across hospitals and teams), we used Huber-White clusterrobust variation estimates.

To analyse differences in mortality rates by hospital and surgical team volume, we included dichotomous $(0 / 1$; no/ yes) variables for low and high volumes (using the medium volume category as reference). The adjusted in-hospital mortality rates for each volume group and corresponding 95\% CIs are presented. Hospital and surgical team volume analyses are reported separately. Additionally, in a combined analysis, we present mortality rates for low, medium and high volume surgical teams who are based in low, medium and high volume hospitals (obtained as the interaction terms of surgical team and hospital volume).

\section{Approval}

The study was endorsed within the NQAIS Clinical Governance Framework. Data on individual hospitals and surgical teams are presented in an anonymised fashion to ensure confidentiality.

\section{Patient and public involvement}

No patient involvement.

\section{RESULTS}

\section{Descriptive analysis}

During the study period (2014-2018), 10344 laparotomies were performed at 24 public Irish hospitals. A slight majority $(52.2 \%)$ of patients were female, $15.5 \%$ were over 80 years of age and $21.7 \%$ had a CCI $>10$. The demographics and clinical characteristics of the included patients are provided in table 1.

The in-hospital mortality rate was 77 per 1000 episodes $(\mathrm{n}=798)$. The 7-day and 30-day in-hospital mortality was 273
(26/1000 episodes) and 595 (58/1000 episodes), respectively. Adjusted in-hospital mortality rates by different patient categories are provided in table 2. In-hospital mortality increased with increasing age: patients aged $>80$ years had an in-hospital mortality of 149/1000 episodes compared with a rate of 12/1000 episodes in patients aged 17-29 years. There was a clear association between the in-hospital mortality rate and the number of comorbidities documented: 96/1000 episodes for patients with a CCI of 4-6; 134/1000 episodes for patients with a CCI >10.

\section{Volume analysis}

Table 3 summarises adjusted in-hospital mortality rates per 1000 episodes, classified by hospital volume, surgical team volume and surgical teams volumes combined with hospital volume. Unadjusted mortality according to hospital and surgeon team volume groups is provided in online supplementary table S3. Of the hospital episodes ( $\mathrm{n}=10344), 1450$ were performed at low volume hospitals (under the principal care of 100 clinical teams), 3069 were performed at medium volume hospitals (under the care of 187 teams) and 5825 were performed at high volume hospitals (under 433 teams). These episodes were performed by surgeons both on patients under their own care and on patients primarily registered under other clinicians. This explains the total number of clinical teams (720).

Analysis of in-hospital mortality by hospital volume did not identify statistically significant differences as the $95 \%$ CIs for the adjusted in-hospital mortality rates for the three volume groups were overlapping.

For analysis of surgical volume, 870 episodes were excluded as the principal surgical team and the procedure team were discordant; 516 episodes were excluded as the procedure team code was not a general surgeon. This reduced the number of teams from 720 to 207. A total of 8958 episodes were included, of which 1042, 2775 and 5141 related to low, medium and high volume teams, respectively. The HRs for 7-day, 30-day and total in-hospital mortality for low surgical volume teams compared with high surgical volume teams were 1.48 (95\% CI 1.09 to 2.01), 1.54 (95\% CI 1.27 to 1.87 ) and 1.82 (95\% CI 1.56 to 2.14 ), respectively. There was a similar difference in mortality between medium and high volume teams.

Regarding the combined analysis of surgical team and hospital volume, in low volume hospitals, there was a total of 33 surgical teams (9 low volume, 16 medium volume and 8 high volume teams), responsible for a total of 1270 discharges. Low volume surgical teams at both low and high volume hospitals had consistently higher mortality than high volume surgical teams but this difference was only statistically significant for high volume hospitals.

\section{DISCUSSION}

This national analysis of EAS in Irish public hospitals establishes an overall in-hospital mortality rate of 76 per 1000 discharges, a rate that is comparable to others reported in the literature. A similar study from Australia showed an in-hospital mortality rate of 88 per $1000 .{ }^{13} \mathrm{An}$ 
Table 1 Descriptive analysis of episodes with 7-day, 30-day and all-time mortality (number of hospital episodes, \%)

\begin{tabular}{|c|c|c|c|c|c|c|c|}
\hline \multirow{2}{*}{$\begin{array}{l}\text { Variable/year } \\
\mathrm{N}\end{array}$} & \multirow{2}{*}{$\begin{array}{l}\text { All episodes } \\
10344\end{array}$} & \multicolumn{2}{|c|}{ Died within 7 days } & \multicolumn{2}{|c|}{ Died within 30 days } & \multicolumn{2}{|c|}{ Died at hospital } \\
\hline & & 273 & $2.6 \%$ & 595 & $5.8 \%$ & 798 & $7.7 \%$ \\
\hline \multicolumn{8}{|l|}{ Sex } \\
\hline Male & 4944 & 125 & $2.5 \%$ & 285 & $5.8 \%$ & 395 & $8.0 \%$ \\
\hline Female & 5400 & 148 & $2.7 \%$ & 310 & $5.7 \%$ & 403 & $7.5 \%$ \\
\hline \multicolumn{8}{|l|}{ Age (years) } \\
\hline $17-29$ & 710 & 2 & $0.3 \%$ & 4 & $0.6 \%$ & 6 & $0.8 \%$ \\
\hline 30-39 & 914 & 3 & $0.3 \%$ & 9 & $1.0 \%$ & 12 & $1.3 \%$ \\
\hline $40-49$ & 1175 & 14 & $1.2 \%$ & 17 & $1.4 \%$ & 24 & $2.0 \%$ \\
\hline $50-59$ & 1593 & 19 & $1.2 \%$ & 38 & $2.4 \%$ & 57 & $3.6 \%$ \\
\hline $60-69$ & 2055 & 49 & $2.4 \%$ & 100 & $4.9 \%$ & 131 & $6.4 \%$ \\
\hline $70-79$ & 2292 & 91 & $4.0 \%$ & 194 & $8.5 \%$ & 273 & $11.9 \%$ \\
\hline $80+$ & 1605 & 95 & $5.9 \%$ & 233 & $14.5 \%$ & 295 & $18.4 \%$ \\
\hline \multicolumn{8}{|l|}{ Admission source } \\
\hline Home & 9562 & 239 & $2.5 \%$ & 521 & $5.4 \%$ & 704 & $7.4 \%$ \\
\hline Other hospitals & 597 & 20 & $3.4 \%$ & 44 & $7.4 \%$ & 61 & $10.2 \%$ \\
\hline Nursing homes & 185 & 14 & $7.6 \%$ & 30 & $16.2 \%$ & 33 & $17.8 \%$ \\
\hline \multicolumn{8}{|c|}{ Charlson Comorbidity Index } \\
\hline 0 & 6591 & 116 & $1.8 \%$ & 183 & $2.8 \%$ & 224 & $3.4 \%$ \\
\hline $1-3$ & 413 & 15 & $3.6 \%$ & 27 & $6.5 \%$ & 34 & $8.2 \%$ \\
\hline $4-6$ & 514 & 26 & $5.1 \%$ & 54 & $10.5 \%$ & 66 & $12.8 \%$ \\
\hline $7-9$ & 577 & 24 & $4.2 \%$ & 54 & $9.4 \%$ & 74 & $12.8 \%$ \\
\hline $10+$ & 2249 & 92 & $4.1 \%$ & 277 & $12.3 \%$ & 400 & $17.8 \%$ \\
\hline \multicolumn{8}{|l|}{ Procedure complexity } \\
\hline Intermediate & 740 & 6 & $0.8 \%$ & 13 & $1.8 \%$ & 14 & $1.9 \%$ \\
\hline Major & 7725 & 207 & $2.7 \%$ & 445 & $5.8 \%$ & 599 & $7.8 \%$ \\
\hline Complex major & 1879 & 60 & $3.2 \%$ & 137 & $7.3 \%$ & 185 & $9.8 \%$ \\
\hline \multicolumn{8}{|l|}{ Organ system } \\
\hline Abdominal wall & 592 & 5 & $0.8 \%$ & 10 & $1.7 \%$ & 11 & $1.9 \%$ \\
\hline Colorectal & 9591 & 267 & $2.8 \%$ & 584 & $6.1 \%$ & 786 & $8.2 \%$ \\
\hline General & 161 & 1 & $0.6 \%$ & 1 & $0.6 \%$ & 1 & $0.6 \%$ \\
\hline \multicolumn{8}{|l|}{ Primary diagnosis } \\
\hline Gastrointestinal & 2443 & 38 & $1.6 \%$ & 83 & $3.4 \%$ & 115 & $4.7 \%$ \\
\hline General & 2792 & 163 & $5.8 \%$ & 283 & $10.1 \%$ & 373 & $13.4 \%$ \\
\hline Hernia/Obstruction & 3025 & 43 & $1.4 \%$ & 117 & $3.9 \%$ & 144 & $4.8 \%$ \\
\hline Malignancy & 2015 & 26 & $1.3 \%$ & 105 & $5.2 \%$ & 152 & $7.5 \%$ \\
\hline
\end{tabular}

English study, based on an analogous database (Hospital Episode Statistics), reported an unadjusted 30-day mortality rate of 136 per $1000 .^{7}$ The National Emergency Laparotomy Audit in England and Wales demonstrated an overall 30-day mortality rate of 106 per $1000 .{ }^{20}$ Analysis of a Danish administrative database including 2904 cases demonstrated a 30 -day mortality rate of 185 per $1000 .{ }^{10}$ Higher mortality rates reported in the UK and in Europe may relate to a different mortality measure (in-hospital mortality not overall 30-day mortality), and different demographics or case-mix.
The overall in-hospital mortality rate conceals important differences between centres and individual surgical teams that are observed in our volume and outcome analysis. We demonstrate a considerable survival advantage when patients are managed by high volume surgical teams. The relationship between outcome and hospital volume is less clear, possibly related to the spread of high, medium and low volume teams across all hospital types. To our knowledge, this is the first study to demonstrate an association between surgeon volume and mortality outcomes in EAS surgery. 
Table 2 Adjusted rates of death per 1000 hospital episodes (95\% Cl)

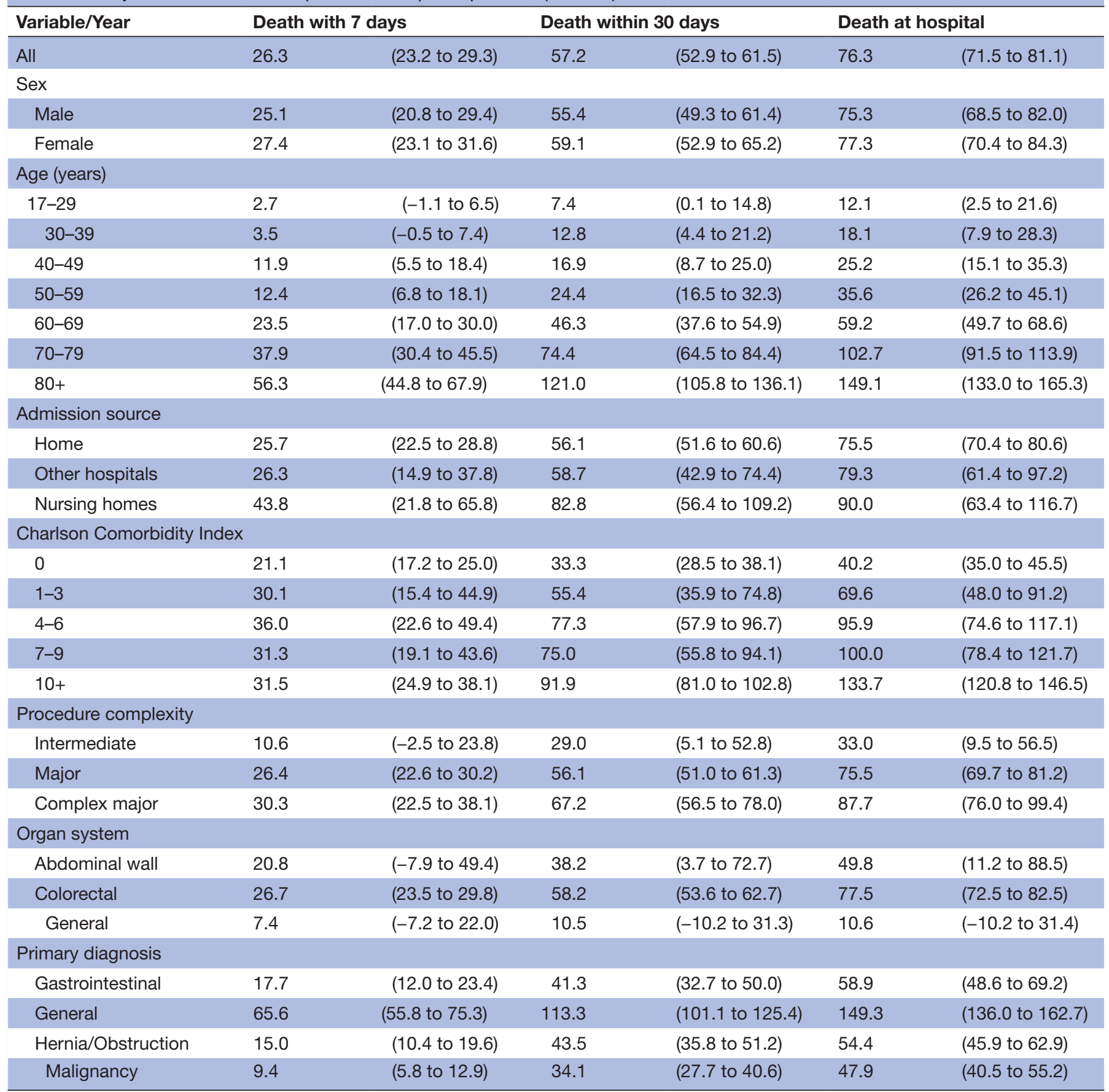

There was no statistically significant association between hospital volume and overall in-hospital mortality demonstrated. Similarly, a comparison of EAS outcomes between patients in England and the USA using administrative datasets ${ }^{7}$ showed no difference in 30-day mortality based on volume. However, the Dr Foster Global Comparators Network database, which accumulates data from 23 large hospitals in the USA, the UK and Australia, demonstrates conflicting results. ${ }^{21}$ In a series of 69490 emergency general surgery (EGS) patients, an overall 30-day mortality rate of 6 per 1000 was documented in the operative subgroup. Hospitals were classified into low volume (<3000 admissions), middle volume (3000-4000) and high volume $(>4000)$ units based on the number of EGS admissions. For patients undergoing surgery, 7-day mortality was $18 \%$ better in middle volume units when compared with low volume centres (30-day mortality OR $0.82,95 \%$ CI 0.68 to $0.99(\mathrm{p}=0.03))$.

In contrast to hospital volume, our study demonstrates that surgeon volume influences in-hospital mortality: EAS performed by surgical teams with low volumes is associated with higher in-hospital mortality than EAS performed by high volume teams. This observation is independent of individual hospital volume. 
Table 3 Adjusted mortality rates per 1000 hospital episodes by volume of hospital and surgical team (95\% Cl)

\begin{tabular}{|c|c|c|c|c|c|c|c|c|}
\hline \multirow{2}{*}{ Hospital volume ( $n=1034$} & \multirow[t]{2}{*}{ Episodes } & \multirow[t]{2}{*}{ Teams } & \multicolumn{2}{|c|}{$\begin{array}{l}\text { Death within } 7 \text { days } \\
\text { postadmission }\end{array}$} & \multicolumn{2}{|c|}{$\begin{array}{l}\text { Death within } 30 \text { days } \\
\text { postadmission }\end{array}$} & \multicolumn{2}{|c|}{ Death at hospital } \\
\hline & & & & & & & & \\
\hline Low $(<250)$ & 1450 & 100 & 25.8 & (17.8 to 33.8 ) & 53.5 & (42.3 to 64.7 ) & 74.2 & (61.1 to 87.2$)$ \\
\hline Medium (250-449) & 3069 & 187 & 24.9 & (19.3 to 30.4$)$ & 62.5 & (54.1 to 70.9 ) & 78.4 & (69.2 to 87.6$)$ \\
\hline High (450+) & 5825 & 433 & 27.2 & (23.0 to 31.4 ) & 55.5 & (49.8 to 61.2$)$ & 75.8 & (69.4 to 82.2$)$ \\
\hline \multicolumn{9}{|l|}{ Team volume $(\mathrm{n}=8958)$} \\
\hline Low $(<30)$ & 1042 & 85 & 30.5 & (20.7 to 40.3 ) & 62.1 & (48.6 to 75.7 ) & 84.5 & (69.0 to 100.0 ) \\
\hline Medium (30-59) & 2775 & 68 & 31.8 & (25.7 to 37.8$)$ & 63.2 & (54.9 to 71.5 ) & 75.1 & (66.2 to 84.0$)$ \\
\hline High (60+) & 5141 & 54 & 18.8 & (14.9 to 22.7 ) & 43.0 & (37.4 to 48.6 ) & 55.0 & (48.8 to 61.1$)$ \\
\hline \multicolumn{9}{|c|}{$\begin{array}{l}\text { Teams at low volume hospitals } \\
(<250)\end{array}$} \\
\hline Low $(<30)$ & 103 & 9 & 36.3 & (0.1 to 72.5 ) & 71.0 & (25.0 to 117.0 ) & 81.9 & (31.4 to 132.4 ) \\
\hline Medium (30-59) & 587 & 16 & 24.7 & (12.5 to 36.9$)$ & 47.6 & (30.9 to 64.3 ) & 60.0 & (41.2 to 78.9 ) \\
\hline High (60+) & 580 & 8 & 18.8 & (8.0 to 29.7 ) & 44.4 & (28.2 to 60.6$)$ & 62.2 & (43.4 to 81.1$)$ \\
\hline \multicolumn{9}{|c|}{ Teams at medium volume hospitals (250-449) } \\
\hline Low $(<30)$ & 344 & 25 & 17.5 & (4.6 to 30.3 ) & 49.6 & (29.2 to 69.9 ) & 78.0 & (53.1 to 102.9 ) \\
\hline Medium (30-59) & 906 & 24 & 28.9 & (18.8 to 39.0 ) & 71.2 & (56.3 to 86.2 ) & 79.4 & (63.7 to 95.2$)$ \\
\hline High (60+) & 1497 & 19 & 23.4 & (15.5 to 31.3 ) & 52.6 & (41.1 to 64.2$)$ & 61.7 & (49.5 to 74.0 ) \\
\hline \multicolumn{9}{|c|}{ Teams at high volume hospitals (450+) } \\
\hline Low $(<30)$ & 595 & 54 & 37.8 & (23.4 to 52.1$)$ & 69.1 & (49.8 to 88.3$)$ & 89.5 & (68.1 to 110.9 ) \\
\hline Medium (30-59) & 1282 & 39 & 36.6 & (27.3 to 45.9$)$ & 64.4 & (52.4 to 76.4$)$ & 78.4 & (65.6 to 91.3$)$ \\
\hline High (60+) & 3064 & 29 & 16.4 & (11.6 to 21.3 ) & 37.9 & (30.9 to 44.8 ) & 50.1 & (42.4 to 57.9 ) \\
\hline
\end{tabular}

Two distinct groups require particular consideration: high volume surgeons in low volume hospitals and low volume surgeons in high volume hospitals. Although high volume surgeons are more likely to work in high volume institutions (29 high volume teams at high volume centres, 8 high volume teams at low volume hospitals; table 3), some surgeons in low volume hospitals perform a similar or greater number of EAS per annum than colleagues in high volume centres due to more frequent on-call commitments. These surgeons can achieve comparable mortality outcomes despite working in lower volume hospitals. In contrast, patients of low volume surgeons in higher volume centres had relatively poorer outcomes despite the availability of enhanced perioperative support and salvage services that are demonstrated to improve outcomes. ${ }^{22}$

The mechanisms behind these volume-outcome relationships are complex and beyond the scope of this national-level analysis. Specialisation of EAS surgeons may be relevant: a survival advantage and improved outcomes are reported when emergency colorectal surgery is performed by colorectal specialists. ${ }^{23}{ }^{24}$ It is also possible that variation in case selection between surgeons exists, although our data fail to demonstrate differences between patients based on available measures of comorbidity.

The heterogeneous outcomes observed in high volume hospitals require attention and highlights the importance of effective clinical governance of emergency abdominal surgery. At the hospital level, recognised factors including perioperative care pathways, timely availability of senior decision-makers, emergency theatre access, critical care resources and access to interventional radiology influence outcome. ${ }^{85-27}$ When a volume-outcome link in elective surgery was identified by Birkmeyer et al, ${ }^{4}$ 'greater transparency and quality improvement...including routine monitoring of surgical performance' was recommended. ${ }^{28}$ Similarly, this study emphasises the need for effective governance for EAS. National audits implemented in the $\mathrm{UK}^{8}$ and Australia ${ }^{29}$ reduced mortality rates in emergency general surgery and a series of quality benchmarks or key performance indicators have been proposed. ${ }^{30}{ }^{31}$ Implementation of a prospective, NELA-like audit process could allow for analysis of specific factors contributing to improved outcomes in high-volume hospitals. However, this would be both financially and logistically difficult in the Irish context.

Centralisation of EAS services has occurred to a variable extent in the Irish health service with higher-risk patients being transferred to larger hospitals in some, but not all, hospital groups. Our health system lacks unique patient identifiers, and so, one limitation of our dataset is incomplete information about transferred patients. The greater mortality previously reported ${ }^{32}$ among transferred patients may improve observed outcomes among lower volume hospitals while adversely impacting in higher volume institutions. However, our observations 
suggest that reducing variation in EAS outcomes nationally requires both national and hospital-based interventions. A simplistic approach of centralising EAS to high volume hospitals is unlikely to improve outcomes unless it is allied to other interventions, including enhanced clinical governance or altered work patterns among surgeons, at high volume institutions.

The strength of this study is the analysis of a complete national EAS database over a contemporary 5-year period. Other studies are limited to selected hospitals ${ }^{721}$ or geographical regions ${ }^{23}$ and may not be generalisable to national populations. Our publicly funded EAS service is available to all residents independent of financial means, reducing variation in access observed in other settings. ${ }^{2633}$ A detailed description of the structure of the health service and the database is provided to allow contextualisation and interpretation for international comparison. The limitations of this study are those inherent to the interpretation of administrative databases. Data accuracy is critically dependent on coding performed at hospital level from clinical records. HIPE consists of administrative data captured at the time of discharge, not clinical data. It captures only in-hospital deaths and hence, probably underestimates overall mortality. HIPE does not contain present-on-admission (POA) flags for secondary diagnoses. Thus, similar to other studies, ${ }^{7}$ mortality was adjusted according to CCI based on comorbidities reported in the medical chart. Acute severe conditions, including complications, can contribute to a high CCI and theoretically mask differences between patients.

\section{CONCLUSION}

EAS patients under the care of high volume surgeons are more likely to survive. Although high volume surgeons more frequently practice in high volume hospitals, the observed higher survival rate is not associated with hospital volume. Reconfiguration of EAS services and more effective clinical governance may reduce the observed variation in mortality.

Acknowledgements The authors would like to thank the Healthcare Pricing Office as the source of HIPE data, which is used in NQAIS Clinical. The authors would like to thank the Clinical Leads of the National Clinical Programmes (National Clinical Programme in Surgery), the NQAIS Clinical Steering Group, the HORC-NCP research group and the Acute Hospital Division (HSE) for providing access to NQAIS Clinical.

Contributors All authors provided a substantial contribution to this project. All authors were involved in conception and execution of the study including identifying the objectives and methodology of the study. DMcN and DOK identified the extraction principles (relevant diagnoses and procedure codes). LH extracted the data from NQAIS. DMcN coded the diagnosis and procedure codes. JS and GV conducted the analyses. DMcN, DOK, JS, KM, GV and DMcN drafted the manuscript. All authors commented on draft versions and approved of the final submission.

Funding The authors have not declared a specific grant for this research from any funding agency in the public, commercial or not-for-profit sectors.

Competing interests None declared.

Patient consent for publication Not required.

Ethics approval Ethical approval was granted by the Research Ethics Committee of the Royal College of Surgeons of Ireland (REC001534).
Provenance and peer review Not commissioned; externally peer reviewed.

Data availability statement № data are available.

Open access This is an open access article distributed in accordance with the Creative Commons Attribution Non Commercial (CC BY-NC 4.0) license, which permits others to distribute, remix, adapt, build upon this work non-commercially, and license their derivative works on different terms, provided the original work is properly cited, appropriate credit is given, any changes made indicated, and the use is non-commercial. See: http://creativecommons.org/licenses/by-nc/4.0/.

\section{ORCID iDs}

Deirdre M Nally http://orcid.org/0000-0001-8590-0021

Gintare Valentelyte http://orcid.org/0000-0001-9188-3854

\section{REFERENCES}

1 Mclsaac DI, Moloo H, Bryson GL, et al. The association of frailty with outcomes and resource use after emergency general surgery: a population-based cohort study. Anesth Analg 2017;124:1653-61.

2 Shahan CP, Bell T, Paulus E, et al. Emergency general surgery outcomes at safety net hospitals. J Surg Res 2015;196:113-7.

3 Shah AA, Haider AH, Zogg CK, et al. National estimates of predictors of outcomes for emergency general surgery. J Trauma Acute Care Surg 2015;78:482-91.

4 Birkmeyer JD, Stukel TA, Siewers AE, et al. Surgeon volume and operative mortality in the United States. $N$ Engl J Med 2003;349:2117-27

5 Becher RD, Hoth JJ, Miller PR, et al. A critical assessment of outcomes in emergency versus nonemergency general surgery using the American College of surgeons national surgical quality improvement program database. Am Surg 2011;77:951-9.

6 Pearse RM, Moreno RP, Bauer P, et al. Mortality after surgery in Europe: a 7 day cohort study. The Lancet 2012;380:1059-65.10.1016/S0140-6736(12)61148-9

7 Tan BHL, Mytton J, Al-Khyatt W, et al. A comparison of mortality following emergency laparotomy between populations from New York state and England. Ann Surg 2017;266:280-6.

8 Saunders DI, Murray D, Pichel AC, et al. Variations in mortality after emergency laparotomy: the first report of the UK emergency laparotomy network. Br J Anaesth 2012;109:368-75.

9 Vester-Andersen M, Lundstrøm LH, Buck DL, et al. Association between surgical delay and survival in high-risk emergency abdominal surgery. A population-based Danish cohort study. Scand $J$ Gastroenterol 2016;51:121-8.

10 Vester-Andersen M, Lundstrøm LH, Møller MH, et al. Mortality and postoperative care pathways after emergency gastrointestinal surgery in 2904 patients: a population-based cohort study. $\mathrm{Br} \mathrm{J}$ Anaesth 2014;112:860-70.

11 Tengberg LT, Cihoric M, Foss NB, et al. Complications after emergency laparotomy beyond the immediate postoperative period - a retrospective, observational cohort study of 1139 patients. Anaesthesia 2017;72:309-16.

12 Broughton KJ, Aldridge O, Pradhan S, et al. The Perth emergency laparotomy audit. ANZ J Surg 2017;87:893-7.

13 Stevens CL, Brown C, Watters DAK. Measuring outcomes of clinical care: Victorian emergency laparotomy audit using quality investigator. World journal of surgery 2017.

14 Al-Temimi MH, Griffee M, Enniss TM, et al. When is death inevitable after emergency laparotomy? analysis of the American College of surgeons national surgical quality improvement program database. $J$ Am Coll Surg 2012;215:503-11.

15 Spence RT, Hampton M, Pluke K, et al. Factors associated with adverse events after emergency laparotomy in Cape town, South Africa: identifying opportunities for quality improvement. J Surg Res 2016;206:363-70.

16 Mealy K, Keane F, Kelly P, et al. What is the future for general surgery in model 3 hospitals? Ir J Med Sci 2017;186:225-33.

17 Benchimol El, Smeeth L, Guttmann A, et al. The reporting of studies conducted using observational Routinely-collected health data (record) statement. PLoS Med 2015;12:e1001885.

18 Vandenbroucke JP, von Elm E, Altman DG, et al. Strengthening the reporting of observational studies in epidemiology (STROBE): explanation and elaboration. Int J Surg 2014;12:1500-24.

19 Healthcare Pricing Office. Irish coding standards (ICS) version 9B2018. Dublin, Ireland: Healthcare Pricing Office, Health Service Executive, 2018.

20 Team NP. Third patient report of the National emergency laparotomy audit London. RCoA, 2017. 
21 Chana P, Joy M, Casey N, et al. Cohort analysis of outcomes in 69490 emergency general surgical admissions across an international benchmarking collaborative. BMJ Open 2017;7:e014484.

22 Finks JF, Osborne NH, Birkmeyer JD. Trends in hospital volume and operative mortality for high-risk surgery. $N$ Engl $\mathrm{J}$ Med 2011;364:2128-37.

23 Brown LR, McLean RC, Perren D, et al. Evaluating the effects of surgical subspecialisation on patient outcomes following emergency laparotomy: a retrospective cohort study. Int J Surg 2019;62:67-73.

24 Zorcolo L, Covotta L, Carlomagno N, et al. Toward lowering morbidity, mortality, and stoma formation in emergency colorectal surgery: the role of specialization. Dis Colon Rectum 2003;46:1461-7.

25 TeamNELA P. Fourth patient report of the National emergency laparotomy audit. London: NELA.

26 Khubchandani JA, Ingraham AM, Daniel VT, et al. Geographic diffusion and implementation of acute care surgery: an uneven solution to the National emergency general surgery crisis. JAMA Surg 2018;153:150-9.
27 Huddart S, Peden CJ, Swart M, et al. Use of a pathway quality improvement care bundle to reduce mortality after emergency laparotomy. British Journal of Surgery 2015;102:57-66.

28 Kizer KW. The volume-outcome conundrum. N Engl J Med 2003;349:2159-61.

29 Kiermeier A, Babidge WJ, McCulloch GAJ, et al. National surgical mortality audit may be associated with reduced mortality after emergency admission. ANZ J Surg 2017;87:830-6.

30 Sugrue M, Maier R, Moore EE, et al. Proceedings of resources for optimal care of acute care and emergency surgery consensus Summit Donegal Ireland. World Journal of Emergency Surgery $2017 ; 12$.

31 Ingraham A, Nathens A, Peitzman A, et al. Assessment of emergency general surgery care based on formally developed quality indicators. Surgery 2017;162:397-407.10.1016/j.surg.2017.03.025

32 Castillo-Angeles M, Uribe-Leitz T, Jarman M, et al. Transferred emergency general surgery patients are at increased risk of death: a NSQIP propensity score matched analysis. J Am Coll Surg 2019;228:871-7.

33 Khubchandani JA, Shen C, Ayturk D, et al. Disparities in access to emergency general surgery care in the United States. Surgery 2018;163:243-50. 